\title{
A Miniaturized Conductor-Backed Slot-Line Resonator Filter With Two Transmission Zeros
}

\author{
Juseop Lee, Member, IEEE, and Kamal Sarabandi, Fellow, IEEE
}

\begin{abstract}
In this letter, a miniaturized planar two-pole microwave filter with two transmission zeros is presented. The building block of this filter is a high- $Q$ double spiral slot resonator backed by a conductor plane. The provision of a float back conductor plane allows vertical integration of this filter with other passive and active RF electronic components usually found in miniaturized wireless transceivers. The conductor plane also provides a coupling mechanism between the input and output of the filter giving rise to transmission zeros. It is shown that the location of transmission zeros can easily be adjusted and significant out-of-band rejection be achieved using only two miniaturized double-spiral resonators. The design procedure and fabrication of a two-pole filter operating at $2.11 \mathrm{GHz}$ is demonstrated and a very good agreement between measured and simulated results is shown.
\end{abstract}

Index Terms-Elliptic-response, microwave Filter, slot-line resonator, transmission zeros.

\section{INTRODUCTION}

$\mathbf{I}$ N RECENT years significant amount of effort has been devoted towards development of high-performance microwave filters. Particular attention has been given to miniaturization, tunability, low insertion loss characteristic, low cost, and multipassband behavior. Novel topologies employing source-load couplings and nonresonating nodes [1]-[3] have been reported. Also for tunability, varactors have been used in various filter's resonators [4], [5]. The synthesis and realization methods for filters with multiple passband characteristics have also been studied widely [6], [7]. To reduce filter dimensions, lumped elements are often used in miniaturized radio frequency (RF) transceivers. However, it is well known that lumped elements at microwave frequencies have very poor quality factors giving rise to significant insertion loss and poor out-of-band rejection. In addition, filter designers must often times deal with undesirable self-resonance behavior of the lumped elements at microwave frequencies. Recently a method for fabricating high performance planar and highly miniaturized microwave filters has been introduced [8]. These filters used slot-line, as opposed to microstrip line or co-planar waveguide, resonators with a unique dual-sprial miniaturized configuration. It was shown that for a miniaturized transmission line type resonator, slot-line resonators provide the highest $Q$. However, the strong near-fields around each resonator of these filters can easily interact with what might be placed beneath the dielectric layer.

Manuscript received June 2, 2006; revised June 15, 2006. This work was supported by the Korea Science and Engineering Foundation under Grant D00103.

The authors are with the Radiation Laboratory, Department of Electrical Engineering and Computer Science, The University of Michigan, Ann Arbor, MI 48109-2122 USA (e-mail: ifsnow@ieee.org; saraband@eecs.umich.edu).

A color version of Fig. 4 is available online at http://ieeexplore.ieee.org.

Digital Object Identifier 10.1109/LMWC.2006.885612
Although transmission zeros can be introduced using a finite ground plane, it is difficult to control their location.

In order to allow vertical integration of other RF components with this filter, this letter presents a modified structure for the miniaturized double-spiral slot-line resonator filter. This structure is formed by placing a conductor plane beneath the slot-line resonators which can shield the backside of a filter formed by these resonators. The back conductor is also used to obtain a controllable source-load coupling that allows placement of transmission zeros in the filter response.

A two-pole miniaturized slot-line resonator filter with two transmission zeros operating at $2.11 \mathrm{GHz}$ is designed and realized to demonstrate the proposed concept. A very good agreement between the simulated results and the measured ones is shown.

\section{Filter Structure AND Design}

Fig. 1(a) and (b) show the structures of two-pole CPW-fed miniaturized slot-line resonator filters one without and one with a back conductor plane. The performance of the conventional slot-line filter (without the back conductor plane) is affected by components or objects placed underneath the resonator and therefore the filter cannot be integrated into multilayer circuits. Although it is possible to introduce transmission zeros in the filter response their locations are mainly affected by the dimensions of the ground plane of the resonator (top layer) and therefore not easily controllable as the size of the top ground plane is a function of transceiver topology and other design rules. The modified filter structure on the other hand shields the resonators from under layers and provides a way to control the location of transmission zeros. To control the transmission zeros, the resonator ground plane on top is split into two parts with a gap shown in Fig. 1(b).

To understand the behavior of the proposed filter, let us consider the signal flow graph shown in Fig. 2. According to the filter specifications, the transfer function of the filter can be obtained by the proper choice of poles and zeros. The relationship between the poles and zeros of elliptic filters is well understood and can be found in [2]. The transfer function of an $N$ th elliptic filter is given by

$$
t^{2}(s)=\frac{1}{1+\varepsilon^{2} R_{N}^{2}(s)}
$$

where $\varepsilon$ is ripple constant and $R_{N}(s)$ is the characteristic function given by

$$
R_{N}(s)=\frac{\prod_{i=1}^{N}\left(s-s_{p i}\right)}{\prod_{j=1}^{O}\left(s-s_{z j}\right)}
$$




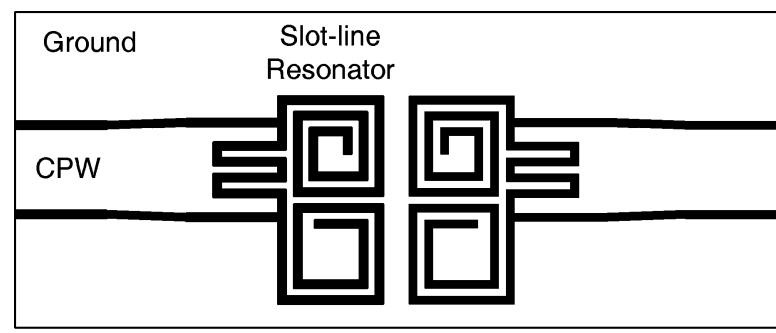

Top View

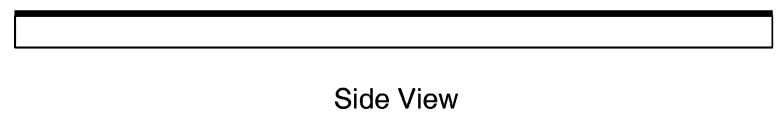

(a)

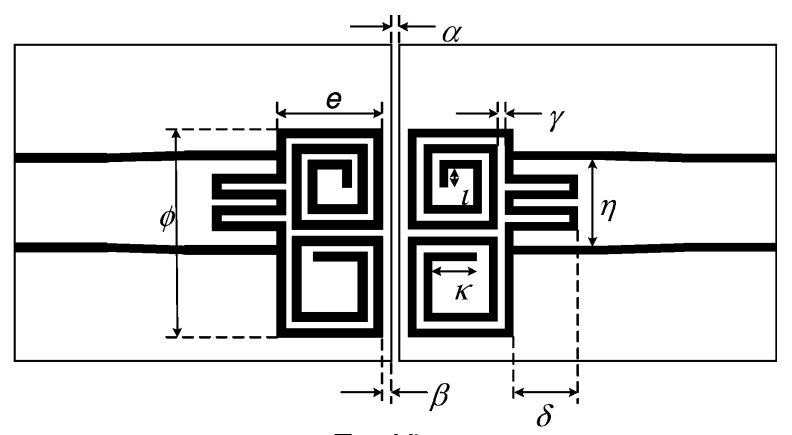

Top View

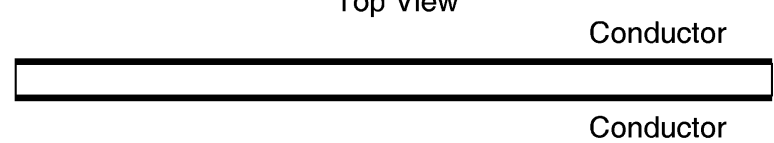

Side View

(b)

Fig. 1. Two-pole miniaturized double-spiral slot-line resonator filters: (a) a conventional structure and (b) a novel structure.

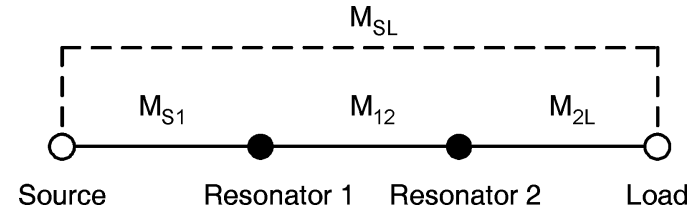

Fig. 2. Signal flow in the two-pole filter with the source-load coupling.

where $s_{p i}$ and $s_{z j}$ are poles and zeros of the filter, respectively. In (2), $O$ is the number of transmission zeros. If the filter has the source-load coupling, $N$ transmission zeros can be realized. In our filter design, the transmission zeros are determined to be at $\pm j 28.0$ with the maximum in-band return loss of $20 \mathrm{~dB}$. From (1) and (2), the transfer function can be rewritten as

$$
t(s)=\frac{1}{\varepsilon} \cdot \frac{s^{2}+a_{z 0}}{s^{2}+a_{p 1} s+a_{p 0}}
$$

where $a_{z 0}=784, a_{p 1}=2.995, a_{p 0}=5.017$ and $\varepsilon=157.073$. Finally, the $(N+2) \times(N+2)$ coupling matrix of the filter can be obtained from (3) and is given by

$$
M=\left[\begin{array}{cccc}
0 & 1.224 & 0 & 0.003 \\
1.224 & 0 & -1.661 & 0 \\
0 & -1.661 & 0 & 1.224 \\
0.003 & 0 & 1.224 & 0
\end{array}\right] .
$$

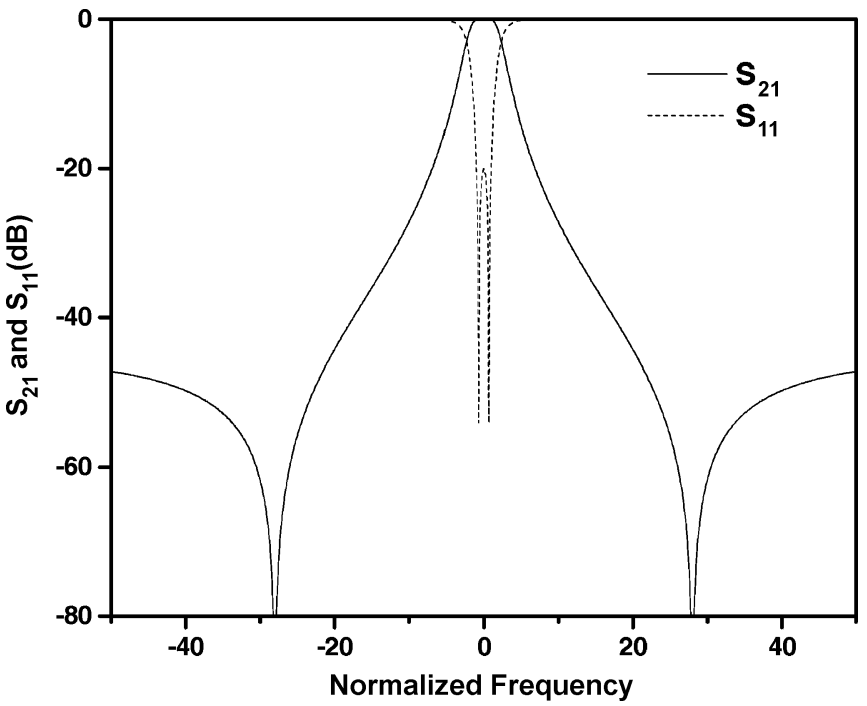

Fig. 3. Frequency response of the filter with the coupling matrix in (4).

TABLE I

DIMENSIONS, IN mm, OF THE FILTER SHOWN IN Fig. 1(b)

\begin{tabular}{|c|c|c|c|c|c|c|c|c|}
\hline$\alpha$ & $\beta$ & $\delta$ & $e$ & $\phi$ & $\gamma$ & $\eta$ & $\mathrm{l}$ & $\kappa$ \\
\hline 0.3 & 0.35 & 2.75 & 4.00 & 7.90 & 0.30 & 3.30 & 0.70 & 1.70 \\
\hline
\end{tabular}

The frequency response of the filter with the coupling matrix in (4) is shown in Fig. 3. The filter is designed to have a center frequency of the $2.11 \mathrm{GHz}$ and a bandwidth of $60 \mathrm{MHz}$.

From the obtained coupling coefficients, the dimensions of the filter can be determined. The coupling coefficient between the source and the load is mainly determined by the distance between the two-ground plane, $\alpha$, and this can be determined by observing the $S_{21}$ response with the following relationship:

$$
S_{21}=\frac{2 M_{S L}}{1+M_{S L}^{2}} .
$$

The external coupling coefficients $\left(M_{S 1}, M_{2 L}\right)$ can be varied by changing the finger length $\delta$ and this can be determined by measuring the phase response of $S_{11}$ with one resonator and using

$$
M_{S 1}^{2}\left(=M_{2 L}^{2}\right)=\frac{\Delta f_{ \pm 90^{\circ}}}{\Delta f}
$$

where $\Delta f$ and $\Delta f_{ \pm 90^{\circ}}$ are the absolute bandwidth of the filter and the frequency difference between two frequencies at which the phase shift $\pm 90^{\circ}$ with respect to the absolute phase at the center frequency $f_{0}[9]$.

The coupling between the resonators is mainly determined by the length $\beta$. Since this coupling cannot be measured without source-load coupling for this filter structure and all the other couplings are known, the length $\beta$ has been determined in the presence of source-load coupling and external coupling by matching the measured pole locations to the theoretical ones.

Using this procedure the filter dimensions shown in Fig. 1(b) have been found and are reported in Table I. All dimensions are in $\mathrm{mm}$ and the slot width for both resonators is a fixed value of $0.30 \mathrm{~mm}$. 


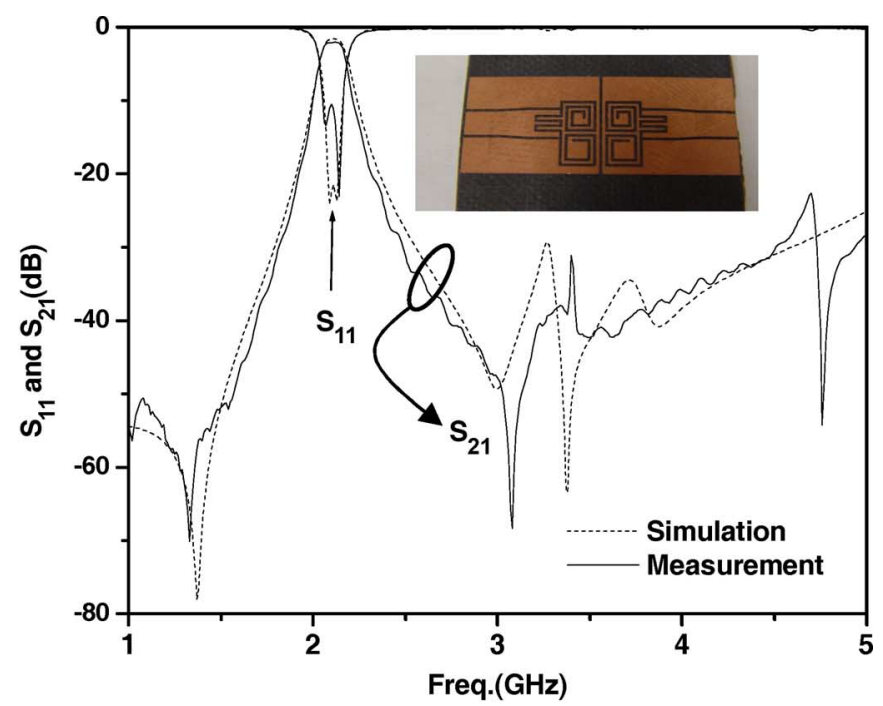

(a)

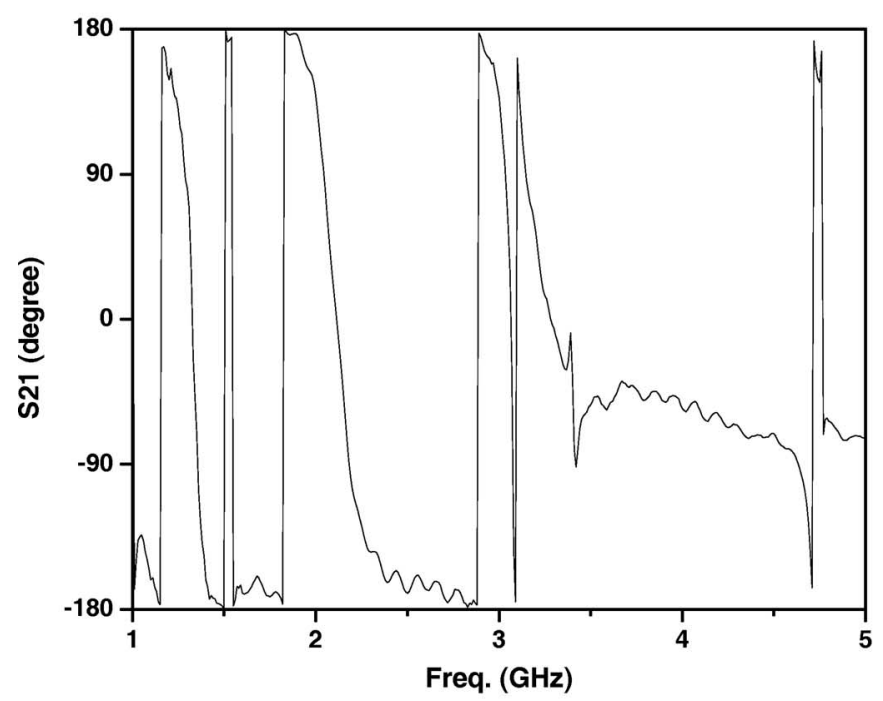

(b)

Fig. 4. Fabricated two-pole slot-line resonator filter and its frequency responses: (a) magnitude of $S_{11}$ and $S_{21}$ and (b) phase of $S_{21}$.

\section{FABRICATION AND MEASUREMENT}

A filter is fabricated on a Duroid 5880 substrate with a thickness of $0.787 \mathrm{~mm}$, dielectric constant of 2.2, and loss tangent of 0.0009. Fig. 4 shows the fabricated filter and compares the measured and simulated responses of the filter. The measured frequency responses of the filter show a good agreement with the simulated results. Note that a two-pole filter can have two transmission zeros and the positions of transmission zeros are nearly identical to each other. The insertion loss has been measured to be $2.0 \mathrm{~dB}$. If the transmission zeros are designed to locate near to the passband, the filter will have higher frequency selectivity.

\section{CONCLUSION}

In this letter, a miniaturized high $Q$ filter with very high selectivity using a conductor plane backed double-spiral slot-line resonator filter is presented. This filter structure allows vertical integration of multilayer circuits and source-load coupling. A prototype miniaturized two-pole filter at $2.11 \mathrm{GHz}$ is fabricated which occupies an area as small as $8 \mathrm{~mm} \times 9 \mathrm{~mm}$ and presents two transmission zeros. The frequency selectivity can be adjusted by controlling the location of the transmission zeros. It is also shown that simulation and measured results agree very well.

\section{REFERENCES}

[1] J. R. Motejo-Garai, "Synthesis of $N$-even order symmetric filters with $N$ transmission zeros by means of source-load cross coupling," Electron. Lett., vol. 36, no. 3, pp. 232-233, Feb. 2000.

[2] S. Amari, U. Rosenberg, and J. Bonermann, "Adaptive synthesis and design of resonator filters with source/load-multiresonator coupling," IEEE Trans. Microw. Theory Tech., vol. 50, no. 8, pp. 1969-1978, Aug. 2002.

[3] S. Amari and U. Rosenberg, "New building blocks for modular design of elliptic and self-equalized filters," IEEE Trans. Microw. Theory Tech., vol. 52, no. 2, pp. 721-736, Feb. 2004.

[4] A. Tombak, J.-P. Maria, F. T. Ayguavives, Z. Jin, G. T. Stauf, A. I. Kingon, and A. Mortazawi, "Voltage-controlled RF filters employing thin-film barium-strontium-titanate tunable capacitors," IEEE Trans. Microw. Theory Tech., vol. 51, no. 2, pp. 462-467, Feb. 2003.

[5] L.-H. Hsieh and K. Chang, "Tunable microstrip bandpass filters with two transmission zeros," IEEE Trans. Microw. Theory Tech., vol. 51, no. 2, pp. 520-525, Feb. 2003.

[6] D. R. Jachowski, "Folded Multiple Bandpass Filter With Various Couplings," U.S. patent 5410284, Apr. 25, 1995.

[7] J. Lee, M. S. Uhm, and I.-B. Yom, "A dual-passband filter of canonical structure for satellite applications," IEEE Microw. Wireless Compon. Lett., vol. 14, no. 6, pp. 271-273, Jun. 2004.

[8] R. Azadegan and K. Sarabandi, "Miniature high-Q double spiral slotline resonator filters," IEEE Trans. Microw. Theory Tech., vol. 52, no. 5, pp. 1548-1557, May 2004.

[9] J.-S. Hong and M. J. Lancaster, Microstrip Filters for RF/Microwave Applications. New York: Wiley, 2001. 